\title{
Research on Edge Detection Algorithm in Digital Image Processing
}

\author{
Zheng Wen-kui*,Liu kun \\ School of Software, Henan University, Kaifeng 475004, China \\ zhengwenkuisja@163.com
}

Key words: Digital image processing; edge detection; algorithm;

\section{Abstract.}

The image has many features, among which the edge is one of the most basic one. The purpose of edge detection is to obtain accurate edge positioning, which plays a vital role in computer vision and image analysis. Great detection of the image can also effectively suppress the generation of noise. There are many methods for edge detection in digital image processing. With the upgrading of science and technology, the recent emerging image edge detection algorithm becomes the organic synthesis of variety emerging image edge detection algorithms. Firstly, the theoretical analysis and LOG operator of the classical edge detection operator are studied emphatically. Then, the performance of the classical edge detection operator and the multi-scale algorithm of the digital image edge detection algorithm are fully explained. Finally, the digital image edge detection classification and digital image edge detection algorithm are described in detail.

Edge detection method refers to a method of segmentation calculation on the edge of an image. The edge detection of the image is also a test of the basic characteristics of the image, and such information detection will carry a lot of image information. Therefore, the image edge detection algorithm plays an important role in the geometric analysis of the image and the application of the computer vision, and can provide a lot of reliable resources for the actual interpretation and data analysis of the image ${ }^{[1-3]}$.

Digital image edge detection algorithm is based on image segmentation, image pixel recognition, partial image acquisition and other image analysis methods. When the image edge detection algorithm is used, it is necessary to perform the edge measurement and edge sampling of the image, so that the image can be understood and the image can be analyzed at the same time. Image edge detection algorithm has become one of the important research fields of image recognition and computer identification. Image edge detection algorithm also plays an important role in engineering application and engineering drawing. Image edge detection algorithm is one of the most basic analysis methods of image. In pursuit of simple algorithms, the image edge detection algorithm also deals with the balance between detection accuracy and noise immunity ${ }^{[4-6]}$. In this paper, the edge detection algorithm in digital image processing is studied deeply, and the conventional classical algorithm is studied. It is hoped that a kind of edge detection algorithm for digital image with simple calculation and balanced detection accuracy and anti-noise can be found.

\section{Theoretical Analysis and Comparison of Classical Edge Detection Operators}

Image edge refers to a collection which contains pixels grayscale with a certain level of change or a set of pixel changes around an image. There is a certain gradation gradient between the two different pixels. The edge feature of the image is one of the most important basic features of the image. Digital image edge can be divided into two categories: the first is the the image step edge, where the pixel gray between images has a significant difference; the second is the roof image edge [7-9].

The edge detection operator realizes the edge detection of the image through the anomaly of 
the edge of the image. This kind of edge detection includes the following types: This kind of edge detection includes the following types: The first edge detection operator using the first derivative method as the calculation method. In the actual calculation process, the method uses $2 \times 2$ or $3 \times 3$ standard template as the core, applies convolution operation to each pixel gray point on image edge, and then selects the appropriate value to calculate the results to provide edge extraction, such as Roberts edge operator, Sobel edge operator, Prewitt edge operator, etc .The second algorithm uses the second derivative as the basis for the operation of the edge detection ${ }^{[10,11]}$. In the calculation process, the number of second derivative zero point is used to calculate the edge detection, such as Laplacian edge operator. The Canny edge operator is an edge detection operator other than the conventional algorithm. The operator does not use the calculus equation to carry out the edge detection, but the edge detection operator is deduced by certain conditional constraints.

For the first derivative edge operator and the edge detection image $Z=f(x, y)$, its pixel gray scale is:

$\mathrm{f}(\mathrm{x}, \mathrm{y})=\left[\begin{array}{l}G_{\mathrm{x}} \\ G_{\mathrm{y}}\end{array}\right]=\left[\begin{array}{l}\frac{\partial \mathrm{f}}{\partial \mathrm{x}} \\ \frac{\partial \mathrm{f}}{\partial \mathrm{y}}\end{array}\right]$

In the formula: $G_{x}$ and $G_{y}$ are pixel gray values. Because the digital image has a certain degree of dispersion, the partial derivative calculation will use the difference method instead of calculus calculation. This can make the calculation easier, you can select the small area of the image, the image convolution can be calculated and estimated, as well as the calculation can be simplified. The three kinds of operators which are often used are: Roberts edge operator, Sobel edge operator and Prewitt edge operator.

The Roberts edge operator is the edge operator calculated through the image local pixel difference, which is obtained by the following equation:

$$
g(x, y)=\left\{[\sqrt{f(x, y)}-\sqrt{f(x+1, y+1)}]^{2}+[\sqrt{f(x+1, y)}-\sqrt{f(x, y+1)}]^{2}\right\}^{\frac{1}{2}}
$$

Select the coordinates for different areas. Using the Roberts operator to perform operations on a $2 \times$ 2 first derivative template, as shown in Figure 1, the convolution kernel in the figure covers the Roberts operator. Each pixel in the image covers two cores as convolutions. The Sobel edge operator is an edge operator computed using a second order function equation, using the pixel difference between the images to calculate the edge operator and then selecting it by the calculated value. It is calculated as follows:

$S=\left(\mathrm{dx}^{2}+\mathrm{dy}^{2}\right)^{\frac{1}{2}}$

The Sobel edge operator is performed on a $3 \times 3$ computational template, as shown in Figure 2, where $\mathrm{dx}$ and dy are the two convolutions of the Sobel edge operator. One core mainly affects the longitudinal edge of the image, and the other has a large impact on the horizontal edge of the image, and the maximum value is chosen as the output value between the two convolutions ${ }^{[12,13]}$. The result of the image operation is an image with a certain edge gray scale. The Prewitt edge operator is the same as the Sobel edge operator. The calculation of the image is done using the selected core for convolution calculation, and also select the maximum value for the output value. It is calculated by the following formula: 


$$
S_{\mathrm{p}}=\left(\mathrm{dx}^{2}+\mathrm{dy}^{2}\right)^{\frac{1}{2}}
$$

It is calculated on a 3x3 template. As shown in Figure 3, dx and dy form the Prewitt operator computing convolution kernel. The Prewitt edge operator eventually produces an image with a certain gray scale on the edge.

\begin{tabular}{|c|c|}
\hline 0 & -1 \\
\hline 1 & 0 \\
\hline
\end{tabular}
operator
Figure 1 Roberts edge

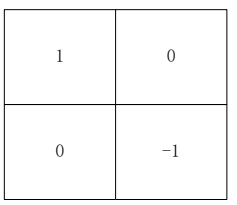

Laplacian edge operator is an edge operator computed by second order calculus. The Laplacian edge operator uses the principle that second derivative function does not intersect at the edge point to perform edge detection. Laplacian edge operator has a certain coherence, it is derived as:

$$
f(x, y)=\frac{\partial^{2}}{\partial_{x}^{2}} f(x, y)+\frac{\partial^{2}}{\partial^{2}} f(x, y)
$$

Since the Laplace edge operator is an vector without normal vector, the Laplace edge operator can only be computed on a template. Unlike the pixel gradient operator, the Laplacian edge operator does not need to calculate the two derivatives. The Laplacian edge operator can be guided by a certain image.

\begin{tabular}{|c|c|c|}
\hline-1 & -1 & -1 \\
\hline-1 & 8 & -1 \\
\hline-1 & -1 & -1 \\
\hline
\end{tabular}

\section{Figure 4 Laplacian edge operator}

Canny edge operator is derived from conventional edge operator and edge detection. It has a certain SNR, and has precise positioning and unilateral calculation principle. The Canny edge operator is calculated as follows: 1 Use the Gaussian equation to calculate the image; 2 uses the first order derivative to calculate the gray scale of the finite difference; 3 apply a certain extreme value operation on gray pixels; 4 Use a certain value to limit the edge value.

$(X, y) * G(x, y, e)$ can be obtained by commutating the image $f(x, y)$ using the Gaussian equation, and then its vector direction and vector size are calculated:

$$
\begin{aligned}
& M=\|f(x, y) * G(x, y, e)\| \\
& A=\frac{f(x, y) * G(x, y, e)}{\|f(x, y) * G(x, y, e)\|}
\end{aligned}
$$

In the formula:G(x,y,e) is variable gauss equation; e is the computing coefficient of the degree of image smoothing. In the actual calculation process, Canny edge operators use multiple fixed values to detect strong edges and weak edges, only when the two edges are in contact with each other, the weaker edge is output, moreover, such operators are not easily changed by noise-signal ratio.

The Oberts edge operator is an edge operator that uses the slope bias to calculate. The value of slope represents the intensity of the operator, and the gradient of the operator is perpendicular to the normal vector. Oberts edge operator has high positioning, but it is easy to cause data loss. Oberts edge operator has no ability to resist SNR because Oberts edge operator does not perform 
correlation smoothing verification. However, the Oberts edge operator has the best effect when dealing with images with high edge steepness .

Both the Sobel edge operator and the Prewitt edge operator are edge operators that use the first order derivative to compute. First of all, these two edge operators need to carry out the corresponding smooth calculation processing. Although the two operators have a certain filtering function, the pixel difference generated between the adjacent pixels has little impact on the Sobel edge operator. The different limits determined by different pixels will generate different Sobel edge operators. These two edge operators have a certain anti-SNR, but can not completely rule out the impact of the existence of SNR on the edge operator. These two edge operators can detect images with a certain SNR, but not suitable for the image with strong and complex SNR.

Canny edge operator is the most excellent operator of edge detection. Canny edge operator has been applied in a lot of image processing and application areas. Canny edge operator processing method is to first select a certain Gaussian equation and smoothing mechanism of the image, and then use the unique calculation method for extreme calculation. After processing the image is converted into a data matrix, and then through limiting the detection value and adjusting the coefficient to achieve the purpose of the edge search. Therefore, Canny edge operator has a strong ability to resist SNR. However, the Canny edge operator sometimes filters out some of the high frequencies, which may cause some loss of data.

\section{LOG operator}

(1) Gaussian equation is used for smoothing. (2) Second-order derivative function is applied to enhance the operator. (3) the basis for edge detection is the number of zero points of the second derivative function and the extreme value of first order derivation. (4) a certain means of calculation is used in the sub-pixel on the basis of edge detection. LOG edge operator is often calculated and tested in a $5 \times 5$ template .

Canny edge operator: Canny edge operator is the most commonly used edge detection operator. The Canny edge operator uses the best smoothing method to filter the signal-to-noise ratio, and then uses the method of calculating the extreme value to substitute the filtered value, so that the image is smoothed and the edge can be found more quickly. Canny edge operator is defined as calculation of the maximum gradient value of pixels. However, the Canny edge operator can not eliminate the SNR and can not process the data accurately. Therefore, the effect of the signal-to-noise ratio must be removed by the high-intensity smoothing calculation, but this calculation increases the difficulty of finding the edge. If we can find a way to effectively improve the sensitivity of the Canny edge operator on the edge, which will play a significant role in promoting Canny edge operator application.

\subsection{Performance Comparison of Classical Edge Detection Operators}

Roberts edge operator is calculated using the first-order calculus function. Edge detection is performed using local pixel difference, and the image with a certain slope can be calculated and detected. The sum of the vectors on the gradient in the image detection process is the slope. Roberts edge operator directly takes the corresponding value into the equation, does not filter the image, and therefore can not rule out the signal to noise ratio factor. Roberts edge operator is suitable for images with small signal-to-noise ratio, and Roberts edge operator is ideal for edge detection of steep edges.

Sobel edge operator and Prewitt edge operator: Both of these edge operators need to filter the image, and then the take image equation to a certain calculation. The difference between the two is only due to the different weights caused by the different degree of image filtering. These two kinds 
of edge operators have better effect on image detection with large signal - to - noise ratio and obvious pixel gradient. Laplacian edge operator is an edge operator computed by second order derivative, where the second derivative function passes the zero point and at the same time the corresponding extreme value is formed with the image. The minimum value of the distance can be used as the coefficient of the image edge detection. Laplacian edge operator extreme value calculation method has the best edge detection effect with image contains certain mutation, and is sensitive to SNR. Laplacian edge operator zero-crossing algorithm can be directly applied on the edge detection of special image, but is limited in a certain scope.

\section{Multi - scale Algorithm for Digital Image Edge Detection Algorithm}

Before the detection, the edge detection of the image is to solve positioning of size and detection of data between the existence of contradictions. In the actual detection process, the image processing often encounter a lot of edge problems, and these problems will directly affect the position of the edge and engineering measurement results. To solve the problem, a method for the detection of image edges is proposed. In this paper, multiple edge detection operators are used to detect the edge of the image, and then the results are compared. This kind of edge detection method has obtained the satisfactory result through the actual calculation and consideration. This approach will fully reflect the results of image detection, and provide a data guarantee for better edge positioning, and accurate mapping measurement .

\subsection{The Optimal Operator for Digital Image Edge Detection Algorithm}

The optimal edge operator also includes many types, which ignores the influence on the signal-to-noise ratio, and makes appropriate adjustments to the data location. With the development of the research field, the image closest to the smoothing function is the Gaussian equation image. The Gaussian function equation is applied to edge detection and edge localization. First, we need to smooth the data, and the data is properly repaired according to the multi-order derivative. This method is called edge modification method.

\section{Conclusion}

In this paper, the edge detection algorithm in digital image processing is researched deeply. Through the research of this paper, we hope to provide a theoretical basis for the edge detection algorithm.

\section{Reference:}

[1] Tsanakas J A, Chrysostomou D, Gasteratos P N B A. Fault Diagnosis of Photovoltaic Modules through Image Processing and Canny Edge Detection on Field Thermographic Measurements[J]. International Journal of Sustainable Energy, 2015, 34(6):351-372.

[2] Xiong Y, Li J, Zuo X, et al. Research on an Edge Detection Algorithm of Remote Sensing Image Based on Wavelet Enhancement and Morphology[J]. Journal of Computers, 2014, 9(5).

[3] Leng X, Ji K, Xing X, et al. Hybrid bilateral filtering algorithm based on edge detection[J]. IET Image Processing, 2016, 10(11):809-816.

[4] Deregeh F, Nezamabadi-Pour H. A new gravitational image edge detection method using edge explorer agents[J]. Natural Computing, 2014, 13(1):65-78.

[5] Xu Q, Varadarajan S, Chakrabarti C, et al. A distributed Canny edge detector: algorithm and FPGA implementation[J]. IEEE Transactions on Image Processing, 2014, 23(7):2944.

[6] Liu J, Tang Q, Yang W, et al. Defects' geometric feature recognition based on infrared image 
edge detection[J]. Infrared Physics \& Technology, 2014, 67:387-390.

[7] Gardiner B, Coleman S A, Scotney B W. Multiscale Edge Detection Using a Finite Element Framework for Hexagonal Pixel-Based Images[J]. IEEE Transactions on Image Processing, 2016, 25(4):1-1.

[8] Zhou S, Zou H, Xing X, et al. A Hybrid Bilateral Filtering Algorithm Based on Edge Detection[J]. Iet Image Processing, 2016.

[9] Chetan S, Seshadri H S, Lokesha V. An Effective Parallelism Topology in Ant Colony Optimization algorithm for Medical Image Edge Detection with Critical Path Methodology (PACO-CPM)[J]. Biophysical Journal, 2015, 3(4):12.

[10] Cao B, Du Y, Xu D, et al. An improved histogram matching algorithm for the removal of striping noise in optical remote sensing imagery[J]. Optik, 2015, 126(23):4723-4730.

[11] Kien L L, Li-Lee C, Chong V C, et al. Automated identification of copepods using digital image processing and artificial neural network[J]. BMC Bioinformatics, 2015, 16(18):S4.

[12] Garcia I, Guzmán - Ramírez E, Pacheco C. CoLFDImaP: A web - based tool for teaching of FPGA - based digital image processing in undergraduate courses[J]. Computer Applications in Engineering Education, 2015, 23(1):92-108.

[13] Liu Y F, Cho S, Spencer B F, et al. Concrete Crack Assessment Using Digital Image Processing and 3D Scene Reconstruction[J]. Journal of Computing in Civil Engineering, 2016, 30(1):04014124. 